\title{
Plasticity and grain boundary motion in vortex matter
}

\section{Paolo Moretti*}

The University of Edinburgh, Center for Materials Science and Engineering, The King's Buildings, Edinburgh EH9 3JL, United Kingdom

E-mail: p.moretti@ed.ac.uk

\section{M.-Carmen Miguel}

Departament de Física Fonamental, Facultat de Física, Universitat de Barcelona, Av. Diagonal 647,E-08028, Barcelona, Spain

\section{Michael Zaiser}

The University of Edinburgh, Center for Materials Science and Engineering, The King's Buildings, Edinburgh EH9 3JL, United Kingdom

\section{Stefano Zapperi}

INFM UdR Roma 1 and SMC, Dipartimento di Fisica, Università "La Sapienza", P.le A. Moro 2, 00185 Roma, Italy

\begin{abstract}
We present an analytical and numerical study of the statistical behaviour of grain boundaries in vortex matter. Dislocation assemblies such as low angle grain boundaries are often observed in this system, determining the emergence of a polycrystalline phase. A theory of vortex polycrystals is proposed, in the conceptual framework of grain boundary pinning. Several aspects, including grain growth, transport properties and hysteretic behaviour are investigated. Results are found in agreement with numerical simulations and experimental observations.
\end{abstract}

International conference on Statistical Mechanics of Plasticity and Related Instabilities August 29-September 2, 2005

Indian Institute of Science, Bangalore, India

\footnotetext{
${ }^{*}$ Speaker.
} 


\section{Introduction}

Magnetic fields play an essential role in the behaviour of superconducting materials. Beyond the well known diamagnetic regime obtained for low magnetic inductions (Meissner effect), the superconductive state is either broken (Type I superconductors) or characterised by a mixed phase (Type II superconductors). As first discussed by Abrikosov [1], in the mixed phase magnetic flux is quantised and carried by vortex lines which are arranged in the form of a triangular lattice. The scientific breakthrough that came along with the discovery of high temperature superconductors (HTSC or high $T_{c}$ materials) has significantly moved the attention of the scientific community towards properties of the mixed phase, as most high $T_{c}$ materials behave in a magnetic field as Type II superconductors.

It is now accepted that at low disorder vortices arrange into a topologically ordered phase: the Bragg glass [2]. The existence of this phase, characterised by long distance ordering, slow relaxation, and other glassy features, has been experimentally confirmed [3]. As in conventional matter, strong enough fluctuations destroy long range order: when temperature is raised the vortex lattice melts into a vortex liquid [4]. Typical melting theories are based on phenomenological criteria with disorder [5], or involve dislocation proliferation mechanisms [6].

Fluctuations are also provided by defects that are intrinsically present in these materials. Vortex density grows linearly with the applied magnetic induction. The theory shows that effects of disorder are stronger for high fields [7] and lead to complex glassy phases, often associated by dislocation proliferation phenomena.

At the same time, the observed lattice contains topological defects, such as dislocations and grain boundaries. The latter are the signature of a vortex polycrystal with crystalline domains of different orientations [8]. Vortex polycrystals have been observed in field-cooling experiments, in various superconducting materials such as $\mathrm{NbMo}$ [8], $\mathrm{NbSe}_{2}[9,10,11], \mathrm{BSCCO}$ [12] and YBCO [13]. The grain size is typically found to grow with applied magnetic field [9, 8]. Moreover, two-sided decoration experiments show that the grain boundaries thread the sample from top to bottom [9], i.e., one observes a columnar grain structure. Despite the abundance of experimental observations, there is no detailed theory accounting for the formation of vortex polycrystals.

Properties and behaviour of isolated dislocations in the vortex lattice have been thoroughly investigated in the past $[14,15]$, but the role of grain boundaries has been often overlooked, although they are often encountered in numerical simulations [16, 17]. For instance, the vortex plastic flow in the Corbino disk geometry is characterised by radial grain boundaries sliding in the tangential direction [16]. In addition, recent numerical simulations indicate the presence of an intermediate polycrystalline stage before the melting transition [17]. This suggests that, in some conditions, grain boundaries may play a role in the melting process, as in the theory of grain boundary induced melting of two dimensional crystals [18].

In the following, we introduce grain boundaries in flux line lattices as linear arrays of dislocations and discuss their elastic properties and behaviour in the presence of disorder. We first present an analytical study of vortex polycrystal growth in terms of the competition between elastic properties of grain boundaries and disorder. We discuss the problem of thermally activated creep of grain boundary structures and finally analyse numerically the role of a polycrystalline ordering in the presence of external driving forces induced by currents. 


\section{Elasticity of grain boundaries}

Properties of grain boundaries in vortex matter determine their response to fluctuations induced by disorder, external stresses or temperature. A low-angle grain boundary can be considered as a linear array of dislocations, whose dynamics is ruled by internal stresses. While ideally a grain boundary minimises its energy by remaining flat, the action of external perturbations leads to deformations that can be described by the theory of elasticity. We compute the self-interaction of a deformed grain boundary extending the results obtained for isotropic elasticity [19] to the case of the highly anisotropic vortex lattice.

An effective description of the vortex lattice is provided by its representation as an elastic crystal of flux lines. On large enough length scales, the elastic energy of the vortex lattice can be expressed in terms of the vortex displacement field $\mathbf{u}$ as follows

$$
\mathscr{H}=\frac{1}{2} \int d^{3} r\left[c_{66}(\nabla \mathbf{u})^{2}+\left(c_{11}-c_{66}\right)(\nabla \cdot \mathbf{u})^{2}+c_{44}\left(\partial_{z} \mathbf{u}\right)^{2}\right],
$$

where $c_{11}, c_{44}, c_{66}$ are the local elastic moduli, and the magnetic induction $\mathbf{B}$ is parallel to the $z$ direction. Within this representation, an ideal low angle grain boundary can be described by an infinite periodic array of straight edge dislocations [20] in the vortex lattice oriented along the $z$ axis, spatially arranged along the $y$ axis with an array spacing equal to $D$, and with Burgers vectors b pointing along the $x$ direction. The wandering of the $i$-th dislocation line is given by $X_{i}(z)$, assuming that all displacements take place within glide planes, i.e. the $x z$ plane, so that $X_{i}(z)$ plays the role of the displacement field of the grain boundary as well.

The grain boundary elastic energy can then be obtained [18] by a suitable expansion of Eq. (2.1). The resulting elastic energy which in Fourier space reads

$$
\mathscr{H}_{G B}=\frac{\pi b^{2}}{2 D^{2}} \int \frac{d Q_{y}}{2 \pi} \int \frac{d k_{z}}{2 \pi}\left(2 c_{66}\left|k_{y}\right|+\sqrt{c_{44} c_{66}}\left|k_{z}\right|\right) X\left(k_{y}, k_{z}\right) X\left(-k_{y},-k_{z}\right) .
$$

Equation 2.2 shows that the elastic energy cost of a small deformation $X$ in a grain boundary is proportional to that square of the deformation and has a linear dependence on the modulus of the deformation wave-vector. The different coefficients of $k_{x}$ and $k_{y}$ reflect the anisotropic character of the vortex lattice. However, it is a common procedure to rescale the $y$ coordinate by a factor $\frac{1}{2} \sqrt{\frac{c_{44}}{c_{66}}}[7]$, in order to get an isotropic reference frame. The elastic Hamiltonian thus becomes

$$
\mathscr{H}_{G B}=K \frac{\pi b^{2}}{2 D^{2}} \int \frac{d^{2} k}{(2 \pi)^{2}}|\mathbf{k}| X(\mathbf{k}) X(-\mathbf{k})
$$

being $\mathbf{k}=\left(k_{y}, k_{z}\right)$ and $K=\sqrt{c_{44} c_{66}}$.

In this limit, the same result is obtained as predicted by the isotropic theory [19]. The nonlocal character of the elastic kernel $(\propto k)$ implies that long range interactions between dislocations stiffen the grain boundary, and that a surface tension approximation is not suitable for a correct description of its elastic properties. In the presence of disorder, grain boundaries are expected to roughen less than isolated dislocations. 


\section{Disorder and pinning theories - an overview}

Quenched disorder induces elastic deformations of the grain boundaries and the interplay of elasticity, disorder and driving forces acting on the boundary can be analyzed in the framework of pinning theories [7]. Pinning of isolated dislocations and of linear dislocation arrays in crystalline materials has been investigated in the past [19,21], in order to provide a microscopical explanation of solution hardening. Here we aim to apply our studies on grain boundary pinning [19] to the context of vortex crystals.

As in the case of isotropic crystals [19], vortex grain boundaries are much stiffer than isolated dislocations, possessing a non-local long-range surface tension. However, the description of the interaction between elasticity and disorder that was formulated for grain boundaries in crystalline materials needs to be refined in the case of vortex lattices. The strong pinning approach (holding for low defect concentrations, see Ref. [19]) is substantially unchanged: in the case of dilute pin densities, obstacles interact individually with dislocation lines.

In the case of weak pinning, instead, random forces are mediated by the vortex lattice. Quenched disorder (vacancies or interstitials in the underlying crystalline structure of the superconducting material, and/or substitutional impurities) deforms the vortex lattice inducing random strain fields. The corresponding random stress fields act on vortex dislocations and dislocation arrays, determining their pinning $[6,18]$.

Driving forces for grain boundary motion can be externally induced by a current flowing in the superconductor or internally generated by the ordering process during grain growth [22]. The presence of a driving shear stress $\tau_{\text {ext }}$ gives rise to a to a total driving force per unit length equal to $F_{\text {drive }}=\tau_{\text {ext }} b L / D[23]$.

At low stress grain boundaries are pinned and the depinning stress $\tau_{c}$ can be estimated from conventional scaling arguments [18, 23]. For low values of the stress $\left(\tau_{\mathrm{ext}} \ll \tau_{\mathrm{c}}\right)$ instead, the response of a grain boundary is mainly due to thermally activated motion in a disordered environment [7]. In this case, we expect a highly non-linear creep motion with an average velocity $v \sim \exp \left[-C\left(\tau_{\mathrm{c}} / \tau_{\mathrm{ext}}\right) \mu_{p l} / T\right]$, where $C$ is a constant, and $\mu_{p l}$ is the plastic creep exponent that quantifies the divergence of the energy barriers $U\left(\tau_{\mathrm{ext}}\right) \sim \tau_{\mathrm{ext}}^{-\mu_{p l}}$ separating metastable states.

\section{Strong pinning, grain growth and thermal activation}

Experimental evidence of polycrystalline order in vortex lattices is provided by direct imaging of the lattice structure in Bitter decoration investigations. Here we propose to study the emergence of polycrystalline order from the point of view of grain growth. A typical setup for decoration experiments consists in lowering the temperature after a magnetic field has been applied to the sample at temperatures above the superconducting phase. During this procedure, known as field cooling, magnetic flux is already present in the sample as it is cooled into the mixed superconducting phase. It is thus reasonable to expect that vortices are originally disordered and that, due to their mutual interactions, undergo a local ordering process. During this process, many dislocations annihilate, and most of the remaining dislocations arrange themselves into grain boundaries with various orientations. The growth of crystalline vortex grains is due to the motion of these separating boundaries. The resulting polycrystalline structure has been indeed observed experimentally by means of Bitter 
decorations of both high $[12,13]$ and low $T_{c}[9,10,11]$ superconducting samples. The effect of quenched disorder is to pin the grain boundaries, hindering the growth process. Thus it is important to envisage the growth of vortex polycrystals as the result of the competition between elasticity and disorder.

Grain growth is driven by a reduction in energy: for an average grain size $R$ and straight grain boundaries, the characteristic energy stored per unit volume in the form of grain boundary dislocations is of the order of $\Gamma_{0} / R$, where $\Gamma_{0}$ is the energy per unit area of a grain boundary. Hence, the energy gain achieved by increasing the grain size by $d R$ is $\Gamma_{0} / R^{2} d R$. Physically, the removal of grain boundary dislocations occurs through the motion of junction points in the grain boundary network. As junction points must drag the connecting boundary with them, which may be pinned by disorder, motion can only occur if the energy gain at least matches the dissipative work which has to be done against the pinning forces. The dissipative work per unit volume expended in moving all grain boundaries by $d R$ is $\tau_{\mathrm{c}} b /(D R) d R$, where $\tau_{\mathrm{c}}$ is the pinning force per unit area. Balancing against the energy gain yields the limit grain size

$$
R_{g} \approx \frac{D \Gamma_{0}}{b \tau_{\mathrm{c}}}
$$

Determining the grain size is thus a matter of finding how the critical stress can be expressed in terms of general properties of disordered vortex matter. To proceed, we have to specify the nature of the "disorder". Several assumptions can be made. In the following we will articulate our discussion around the concept of pin density distinguishing weak pinning and strong pinning regimes.

In the weak pinning regime quenched disorder pins the vortex lattice (of lattice constant $a$ ) over a distance $R_{a}$ (Larkin length). Grain boundaries perceive a distribution of stresses due to the elastic straining of the vortex lattice $[6,15]$. Such a distribution acts as a pinning field for the grain boundary and the depinning stress can be computed within the framework of collective pinning theory: the energy associated with bending a grain boundary fraction of linear dimension $L<R_{a}$ over the characteristic distance $X$ can be estimated as (see Reference [23]):

$$
\mathscr{E}=\frac{K b^{2}}{D^{2}} L X^{2}-\frac{K a b}{D} L X\left(\frac{L}{R_{a}}\right)^{1 / 5}+\tau b L^{2} X / D
$$

The first term represents the elastic energy derived in Eq. 2.2, the second the pinning energy [23] and the third the work done by an external driving stress $\tau$ in displacing the boundary. Minimizing the first two terms of Eq. 4.2, for $X=a \simeq b$ [15], we obtain the plastic Larkin length $L_{p} \simeq(b / D)^{5} R_{a}$, which is typically smaller than $R_{a}$. The depinning stress is identified as the stress necessary to depin a section of dimension $L_{p}: \tau_{c}=K b^{2} /\left(D L_{p}\right)$. Combining this expression with Eq. (4.1), using $\Gamma_{0} \simeq K b^{2} / D$, we obtain $R_{g} \sim R_{a}$. The identification of $R_{g}$ with $R_{a}$ was proposed in Ref. [8], but was not confirmed by experiments (see Fig. 1 and Ref. [8]). We therefore propose to interpret the experimental data under a strong pinning assumption.

In this regime dislocations forming the grain boundary are pinned by individual obstacles. We consider here the case of columnar defects, oriented along the $z$ axis. Although not general, this is a sensible assumption, as it holds in a wide variety of experimentally observed vortex arrangements. Its quasi two-dimensional characterisation mimics vortex lattices typically observed 
in highly anisotropic superconductors, where vortices are split onto weakly coupled layers. This is the case, for instance, in the experiments of Ref. [8] where grain boundary pinning is provided by screw dislocations in the superconducting crystal. Under these circumstances, the problem becomes effectively two dimensional and the grain boundary is a one-dimensional string exhibiting long-range elasticity. Hence we can directly apply the strong pinning theory of Friedel [24].

The basic idea is to consider a grain boundary segment as it depins from a pair of strong obstacles. The length $L$ of the segment corresponds to the effective spacing between obstacles along the grain boundary, and it forms a bulge of maximum width $X$. After the grain boundary segment overcomes the pin it will travel by an amount which is, again, of the order of $X$ and, hence, sweep an area of the order of $L X$. At the depinning threshold, the grain boundary starts to move through a sequence of statistically equivalent configurations, and the freed segment will encounter, on average, precisely one new obstacle in the course of this process. This argument leads to the condition $L X \simeq 1 / \rho$ where $\rho$ is the area density of pinning defects. The elastic energy per unit length of the bulge of width $X$ and extension $L$ is $2 c_{66} b^{2} X^{2} / D^{2}$, and should balance the work per unit length $\tau_{\text {ext }} b L X / D$ done by the driving stress $\tau_{\text {ext }}$ in bowing the boundary. This energy balance provides a relation between $L$ and $X$. Furthermore, at depinning the total force $h \tau_{\text {ext }} b L / D$ should be equal to the defect strength $f_{0}$, where $h$ is the sample thickness. Combining the equations above we obtain the depinning stress $\tau_{\mathrm{c}} b=D f_{0} /\left(h L_{f}\right)$, where the Friedel length $L_{f}$ is given by $L_{f}=2 c_{66} b^{2} h /\left(f_{0} \rho D^{2}\right)$. Inserting the expression for the critical stress in Eq. (4.1) together with the scale-independent surface tension $\Gamma_{0}=2 c_{66} b^{2} / D$, we obtain

$$
\frac{R_{g}}{b} \approx \frac{c_{66}^{2} b^{3} h^{2}}{D^{3} f_{0}^{2} \rho} .
$$

In order to use this result to fit the data in Ref. [8], we have to express it in terms of the reduced field $\tilde{B} \equiv B / H_{c 2}$, where $H_{c_{2}}$ is the upper critical field of the superconductor. The field dependence is implicit in the parameters $b$ and $D$, i.e. $b \sim D \sim a \sim \tilde{B}^{-1 / 2}$, as well as in the shear modulus $c_{66} \sim \tilde{B}$, and in the pinning strength $f_{0}$. The pinning force due to a screw dislocation was computed in Ref. [25] and is given by $f_{0} \propto \tilde{B}^{1 / 2}(1-\tilde{B}) \ln \left(\xi_{s c} / 2.7 b_{0} \tilde{B}\right) \approx \tilde{B}^{1 / 2} \ln \left(\xi_{s c} / 2.7 b_{0} \tilde{B}\right)$, where $\xi_{s c} \simeq 100 \dot{A}$ is the coherence length [26], and $b_{0} \simeq 5 \dot{A}$ is the Burgers vector of the screw dislocation [25]. The resulting expression predicts a linear field dependence of the grain size with logarithmic corrections. The agreement with magnetic decoration data from Ref. [8] (Fig. 1) is quite satisfactory, especially if compared to the estimate based on local elasticity assumptions.

Although in field cooling experiments the effects of perturbations due to pinning become predominant, thermal fluctuations could induce an activated motion of the grain boundaries, particularly in high $T_{c}$ materials. This problem can be approached generalising scaling theories of creep for vortices and dislocations [7, 15]. In the weak pinning regime, the relevant energy barrier that the grain boundaries have to surmount under an applied stress $\tau_{\text {ext }}<\tau_{\mathrm{c}}$ is given by $U\left(\tau_{\text {ext }}\right)=U_{0}\left(\tau_{\mathrm{c}} / \tau_{\text {ext }}\right)^{\mu_{p l}}$, where $U_{0} \simeq K b^{3} R_{a}$ and $\mu_{p l}=1$ (Reference [18]). In our case, the applied stress is the ordering stress, so that we have $\tau_{\mathrm{c}} / \tau_{\mathrm{ext}} \simeq R / R_{a}$. Using this expression in the energy barrier for thermally activated grain growth, we obtain

$$
t_{0} \frac{d R}{d t}=R_{a} \exp \left[-\frac{U_{0}}{K_{B} T} \frac{R}{R_{a}}\right]
$$




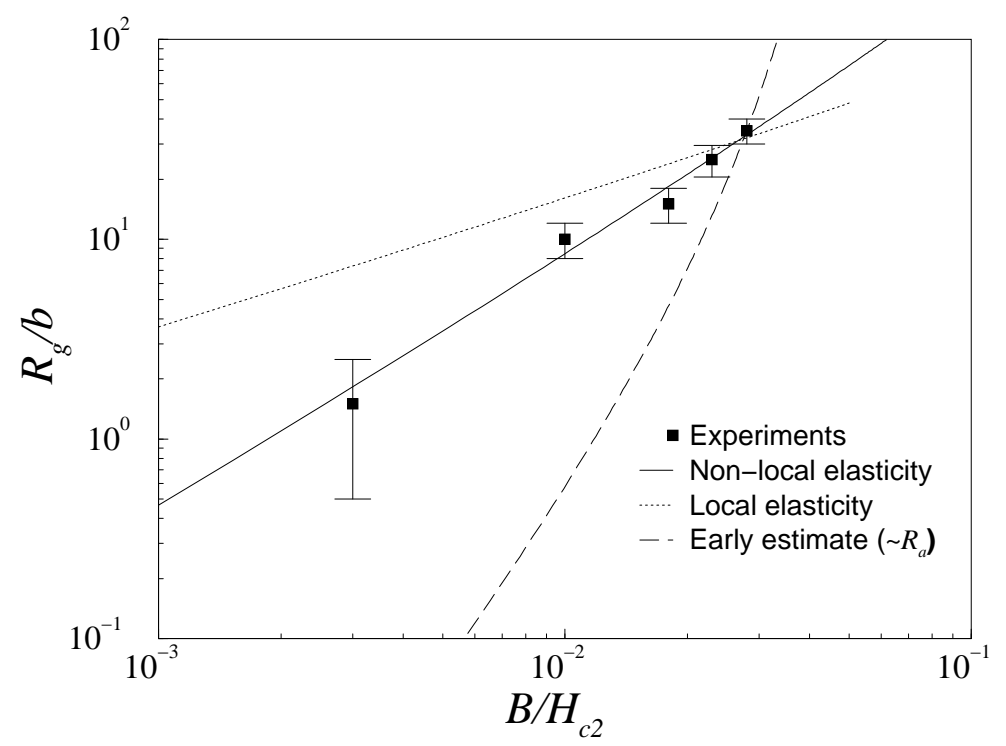

Figure 1: The grain size of a vortex polycrystal experimentally obtained from Bitter decoration of a NbMo sample as a function of the applied magnetic fi eld (Ref. [8]) is compared with the theoretical predictions. For comparison we report as well the result obtained using Friedel theory with local elasticity and the earlier estimate from Ref. [8], formally equivalent to our weak pinning result.

where $t_{0}$ is the appropriate characteristic time. The equation can readily be solved yielding, in the long time limit, a logarithmic growth $R(t) / R_{a}=k T / U_{0} \log \left(t / t_{0}\right)$. This law holds for $R>R_{a}$ when the grain boundaries would be pinned at $T=0$. In the initial growth stage $R \ll R_{a}$, we can neglect pinning forces and the dynamics is ruled by the ordering stress: $\dot{R} \sim 1 / R$, yielding a power law growth $R(t) \sim \sqrt{t}$. Besides thermal activation, grain boundaries can move in response to an applied current [15]. Details on this aspect will be given in the following section.

\section{Effect of grain boundaries on the critical current}

The critical current is an important property of Type II superconductors, since it represents the current below which vortices are pinned and the material conducts without resistance. This technological aspect, however, only partly explains the role of critical current in the system under examination. Externally induced currents produce Lorentz-like driving forces in the flux line lattice. This system can be easily mapped to a general depinning model for a random elastic manifold, where the critical current acts as a depinning force. Such a problem has been analysed in the past and has led to a further broadening of the vortex phase diagram. An exhaustive review can be found in Ref. [7]. Our concern is to point out how this scenario changes in the presence of a polycrystalline arrangement.

In Section 2 we introduced non-local elasticity properties of grain boundaries in vortex matter. This result dramatically influences the behaviour of such systems in the presence of disorder [18]. For instance, it is reasonable to believe that in vortex polycrystals, properties of critical current could change significantly. One can actually expect the critical current to be higher in the presence of grain boundary networks. In order to assess the validity of that statement, we have performed a 

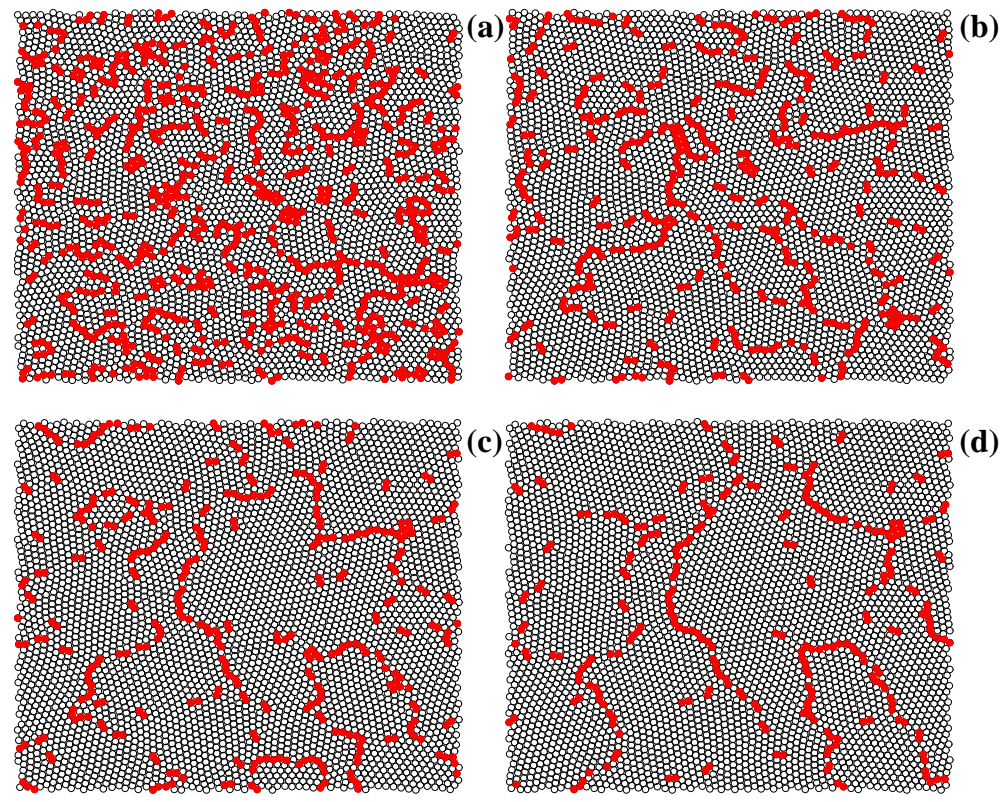

Figure 2: Relaxation of the topological defect structure from a simulation of $N_{v}=4128$ interacting vortices after a sudden fi eld cooling from a disordered vortex state in a simulation cell of linear size $L=36 \lambda_{s c}$. The colored fi ve/seven-fold coordinated vortices (fi lled circles) indicate dislocations in the vortex lattice. The fi nal confi guration (snapshot (d)) is completely pinned by disorder. There one can observe a polycrystalline structure with most dislocations arranged into grain boundaries.

numerical study of the problem. In the following, we will briefly review our results, emphasising how they confirm predictions based on our theoretical model and match the behaviour commonly observed in experiments.

In the simulation of arrays of $N_{v}$ interacting vortices, a polycrystalline vortex structure is obtained by relaxing at zero temperature a random initial vortex arrangement. This process mimics a typical field cooling experiment in which the temperature is rapidly decreased from above $T_{c}$ in presence of a field. The system moves rapidly towards lower energy configurations corresponding to zero temperature and thermal effects can thus be disregarded. In this case magnetic flux is already present in the material in the form of initially disordered vortices. Once grain growth has stopped, the system has a polycrystalline structure and an external current can be simulated by simply applying a constant Lorenz force. The critical current is then defined as the current at which vortices start to move steadily.

During the relaxation process, it is sensible to disregard thermal effects, mimicking the dynamics of the vortex system after a sudden quench of the superconducting sample from high temperatures (or equivalently, random vortex configurations) towards the lower energy states corresponding to zero temperature. After a transient regime, the dynamics stops due to disorder.

The gradual ordering process involves the arrangement of dislocations in grain boundaries (Fig. 2). Long range elastic stress fields are screened and the vortex array rearranges into a polycrystal, which evolves in time until the residual stresses accumulated in the distorted vortex lattice drop down below the critical value $\tau_{\mathrm{c}}$. At this point, grain boundaries get pinned by disorder limiting the average grain size (see Fig. 2(d)). Moreover, the limit grain size $R_{g} / a$ appears to increase 

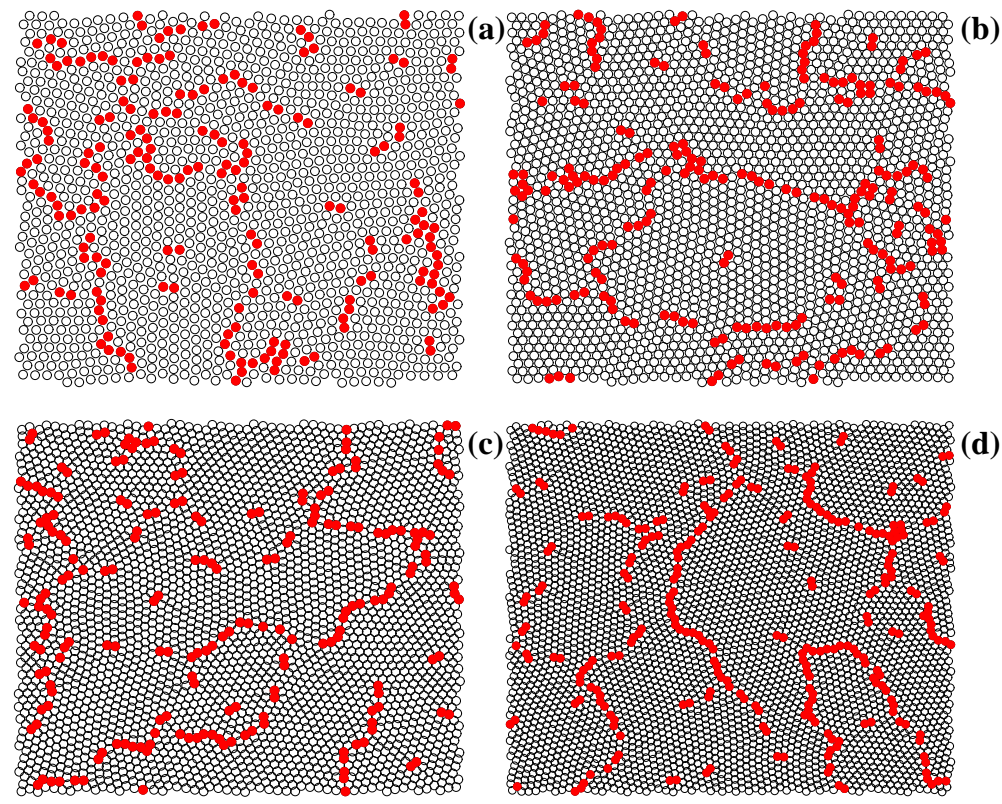

Figure 3: Pinned vortex structure for different values of the magnetic fi eld: (a) $N_{v}=1460$, (b) $N_{v}=2064$, (c) $N_{v}=2919$, (d) $N_{v}=4128$, after a sudden fi eld cooling from a disordered vortex state in a simulation cell of linear size $L=36 \lambda_{s c}$. The colored fi ve/seven-fold coordinated vortices (fi lled circles) indicate dislocations in the vortex lattice. The average grain size in the resulting polycrystalline structure seems to grow with the intensity of the average magnetic fi eld inside the cell.

with magnetic field $B \propto N_{v}$ (see Fig. 3), in qualitative agreement with experimental results [8] and confirming the theoretical predictions reported in Section 4.

Once simulations have clarified the mechanism behind polycrystal formation, one can proceed introducing external forces into the system, in order to investigate the behaviour of the critical current $J_{c}(B)$. An externally applied current may induce the annealing of metastable configurations (see Figs. 2 and 3), resulting in initial transients of plastic flow, which might eventually cease once a new metastable configuration is found, provided that the current is below the threshold value.

Results for the investigation of the dependence of the critical current on the magnetic field are summarized in Fig. 4. Along with the initially polycrystalline arrangement, the study has been also led for a grain boundary-free initial configuration. The qualitative and quantitative differences between the two curves represented in the figure are due to the presence of grain boundaries. The presence of these topological defects in the vortex configuration enhances the critical current needed to give rise to a steady regime of plastic flux flow, that in this case, appears to be controlled by grain boundary motion.

Plastic deformation of crystalline materials is usually mediated by proliferation and motion of dislocations. Nonetheless, another possible mechanism for plastic flow is the glide motion of grain boundaries which, as in this case, can be the most relevant mechanism for small grain sizes, as in the case of nanocrystals.

According to numerical results, grain boundaries are more efficiently pinned by disorder, as single grains are supposed to adjust better to the disordered landscape than a whole perfectly ordered lattice. In both cases, we observe the decrease of $J_{c}$ with an increasing magnetic field (i.e. 
density of vortices) until this reaches a plateau region.

Experiments show that for even higher magnetic fields, the critical current exhibits a sudden increase. Close to the upper critical field $B_{c_{2}}=\Phi_{0} / 2 \pi \xi_{s c}$ ( $\Phi_{0}$ being the magnetic flux quantum), the penetration depth $\lambda_{s c}$ and the coherence length $\xi_{s c}$ are supposed to diverge, and the renormalisation of such parameters is expected to explain that sudden increase [27]. However, the system studied above is far below $B_{c_{2}}$, as we are not concerned about the behaviour in the vicinity of that transition.

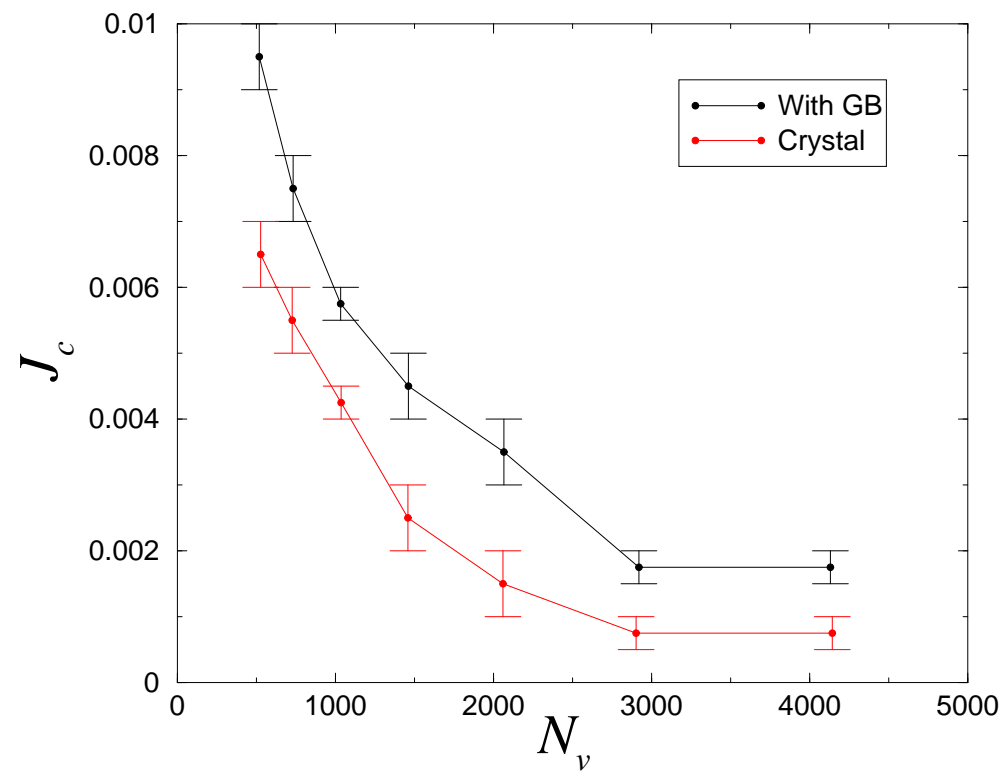

Figure 4: The critical current $J_{c}$ as a function of the number of vortices $N_{v}$ in the simulation cell. The number of pinning points is $N_{p}=4128$, the cell size $L=36 \lambda_{s c}$. The upper line shows the results obtained starting from initial fi eld-cooled confi gurations containing grain boundaries (GB), whereas the lower curve shows the numerical results obtained from perfect crystalline initial confi gurations. Currents are measured in units of $G b^{2} c / \Phi_{0}$.

Regarding experiments, our results match, at least on a qualitative basis, the behaviour exhibited by vortex matter in critical current measurements at low magnetic fields. As stated above, grain boundaries are commonly observed in field-cooled (FC) samples. On the other hand, ordered vortex crystals can be obtained in zero field cooling (ZFC) experiments, i.e. applying a magnetic field only after temperature has been lowered to the expected value [28, 29, 30]. The FC state is usually characterized by a higher critical current and has been proven to be metastable [29, 30]. These aspects result in a peculiar hysteretic behaviour commonly observed in critical current measurements [29, 30] and $I-V$ characteristics [28]. In our numerical analysis, the evaluation of critical currents in perfect vortex crystals (lower line in Fig. 4) fairly mimics the phenomenology of ZFC measurements, while results for the grain boundary model (upper line in Fig. 4) can be interpreted as a simulation of FC response. Hysteresis is in fact reproduced by our simulations when we start from the polycrystalline state. As shown in Fig. 5, when the current $J$ is ramped up vortices start to move at a current $J_{c 1}$, with a velocity that then increases with the current. If the current is ramped down from the moving state, vortices get pinned at a lower value of the current $J_{c 2}$ corresponding to the critical current measured for a perfect crystal upon ramping up the field. Notice the similarity 
with the experimental results of Ref. [28]. Once more, we should underline that these results hold only for low values of the applied field. As the magnetic induction approaches its critical value, a sudden increase in measured critical currents is observed in both the ZFC and the FC experimental setup $[29,30]$.

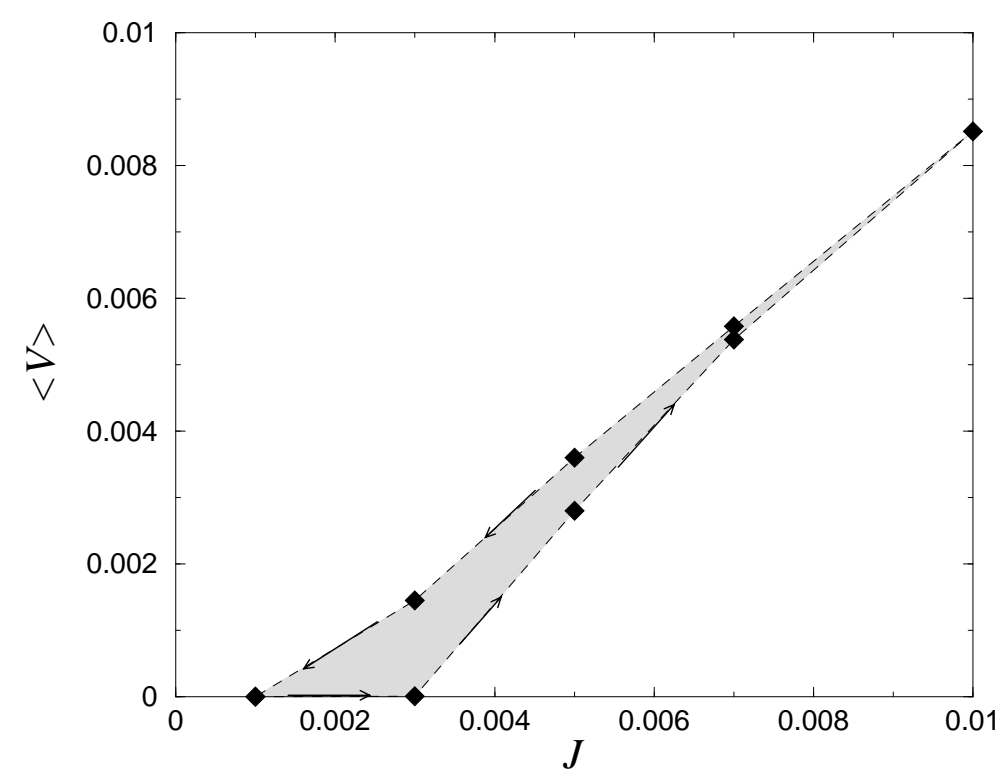

Figure 5: The steady state average velocity of the vortices as a function of the applied current $J$. The current is ramped up (and down) in steps and is kept constant after each step until the system reaches a steady state. The arrows indicate the direction of the ramp. The number of vortices is $N_{v}=2064$, the number of pinning points $N_{p}=4128$, the cell size $L=36 \lambda_{s c}$. Currents are measured in units of $G b^{2} c / \Phi_{0}$.

\section{Conclusions}

Grain boundaries and grain structures are often observed in vortex lattices. However no theory currently accounts for polycrystalline ordering. We derived the exact solution for the elastic problem of a grain boundary in a flux line lattice and formulated a theory of a vortex polycrystal in the presence of disorder. Grain growth is first examined and the derived average grain size is found in good agreement with experiments. Then the problem of transport in the underlying superconductor is addressed. Pinning of topological defects in the vortex lattice is expected to influence the response to an applied current. Currents act in the form of external Lorentz forces driving vortex motion. Below a critical current, the vortex lattice is pinned by disorder and conduction takes place without resistance. Above the critical current, instead, vortices start gliding in the transversal direction, causing dissipation. We found that in the presence of a polycrystalline arrangement, a higher critical current is expected, and the region characterised by zero-resistance is broader. The hysteretical behaviour commonly observed in experiments proves to be a natural consequence of grain boundary depinning. 


\section{Acknowledgment}

Support of EPSRC under Grant No. GR/S20406/01 is gratefully acknowledged.

\section{References}

[1] A. A. Abrikosov, Zh. Eksp. Teor. Fiz. 32, 1442 (1957).

[2] T. Giamarchi and P. Le Doussal, Phys. Rev. B 52, 1242 (1995).

[3] T. Klein, I. Joumard, S. Blanchard, J. Marcus, R. Cubbit, T. Giamarchi, and P. Le Doussal, Nature (London) 413, 404 (2001).

[4] H. Safar et al., Phys. Rev. Lett. 69, 824 (1992).

[5] G. P. Mikitik and E. H. Brandt Phys. Rev. B 68, 054509 (2003).

[6] J. Kierfeld and V. M. Vinokur, Phys. Rev. B 61, 14928

[7] G. Blatter et al., Rev. Mod. Phys. 66, 1125 (1994).

[8] I. V. Grigorieva. Sov. Phys. JETP 69, 194 (1989).

[9] M. Marchevsky, A. Keurentjes, J. Aarts, and P. H. Kes, Phys. Rev. B 57, 6061 (1998).

[10] F. Pardo et al., Phys. Rev. Lett. 78, 4633 (1997).

[11] Y. Fasano et al., Phys. Rev. B 66, 020512 (2002).

[12] H. Dai, J. Liu and M. Lieber, Phys. Rev. Lett. 72, 748 (1994).

[13] J. A. Herbsommer, G. Nieva and J. Luzuriaga, Phys. Rev. B 62, 678 (2000).

[14] M.C. Miguel and M. Kardar, Phys. Rev. B 56, 11903 (1997).

[15] J. Kierfeld, H. Nordborg and V. M. Vinokur, Phys. Rev. Lett. 85, 4948 (2000).

[16] M.-C. Miguel and S. Zapperi, Nature Mat. 2, 477 (2003).

[17] C. Dasgupta and O. T. Valls, Phys. Rev. Lett. 91127002 (2003); Phys. Rev. B 69, 214520 (2004); Phys. Rev. B 72, 094501 (2005).

[18] P. Moretti, M.-C. Miguel, and S. Zapperi, Phys. Rev. B 72, 014505 (2005).

[19] P. Moretti, M.-C. Miguel, M. Zaiser, and S. Zapperi, Phys. Rev. B 69, 214103 (2004).

[20] J.P. Hirth and J. Lothe, Theory of Dislocations, McGraw Hill (1968).

[21] R. Labusch, Phys. Status Solidi 41, 659 (1970); Acta Metall. 20, 917 (1972).

[22] P. M. Hazzledine and R. D. J. Oldershaw, Phil. Mag. A 61, 579 (1990).

[23] P. Moretti, M.-C. Miguel, M. Zaiser, and S. Zapperi, Phys. Rev. Lett. 92, 257004 (2004).

[24] J. Friedel, Dislocations, Pergamon Press (1967).

[25] E. Schneider, J. Low. Temp. Phys. 31, 357 (1978).

[26] G. H. Kruithof, P. C. van Son, and T. M. Klapwijk, Phys. Rev. Lett. 67, 2725 (1991).

[27] M. Chandran, R. T. Scalettar, and G. T. Zimányi, Phys. Rev. B 69, 024526(2004).

[28] Z. L. Xiao et al., Phys. Rev. Lett. 86, 2431 (2001).

[29] W. Henderson et al., Phys. Rev. Lett. 77, 2077 (1996).

[30] S. S. Banerjee et al., Appl. Phys. Lett. bf 74, 126 (1999). 